\title{
ULCERAÇÃO DE ANASTOMOSE ESÔFAGO-ENTÉRICA CAUSADA POR ALENDRONATO ${ }^{+}$
}

\author{
Pedro DUQUES*, Renata Soares Almeida ARAÚJO** e \\ Waldir Pedrosa Dias de AMORIM***
}

\begin{abstract}
RESUMO - Introdução - O alendronato de sódio, um aminobifosfonado indicado para o tratamento da osteoporose em mulheres pós-menopausadas, tem sido associado em vários relatos à esofagite. Estenose esofágica e sintomas gastrointestinais como dispepsia, náuseas, vômitos e dor abdominal também podem estar presentes. Objetivo - Relato do caso de paciente submetida a gastrectomia total com anastomose em $Y$ de Roux devido a carcinóide gástrico que desenvolveu ulceração em anastomose esôfago-entérica após tratamento com esta droga. Material e Método-Paciente de 63 anos de idade, sexo feminino, iniciou terapia com alendronato na dose diária de $10 \mathrm{mg}$ pela manhã, vindo a apresentar sintomas disfágicos e dor abdominal um mês após. Submetida a exame endoscópico, demonstrou-se ulceração no esôfago, na mucosa da anastomose esôfago-entérica e estenose esofágica. O tratamento medicamentoso foi descontinuado, tendo o sintoma de dor abdominal desaparecido e a disfagia diminuído de intensidade. Resultados - Houve remissão das lesões ulceradas, entretanto a estenose esofágica permaneceu. A paciente subseqüentemente foi tratada por dilatação endoscópica, evoluindo com melhora clínica e estando atualmente livre de sintomas. Conclusão - $O$ alendronato de sódio tanto pode causar lesões na porção inferior do esôfago, como em segmentos mais distais do tubo digestivo nos pacientes cujo trânsito gastrointestinal é acelerado. Especial atenção deve ser dada aos pacientes gastrectomizados que fazem uso desta droga, pela possibilidade do desenvolvimento de lesões na mucosa entérica anastomosada e do surgimento de complicações temíveis como a estenose.
\end{abstract}

DESCRITORES - Alendronato. Estenose esofágica. Anastomose em-Y de Roux. Esôfago, cirurgia.

\section{INTRODUÇÃO}

O alendronato de sódio (Fosamax ${ }^{\circledR}$, Merck) é droga inibidora da reabsorção óssea usada no tratamento da osteoporose em mulheres pós-menopausadas ${ }^{(4)}$. O mecanismo pelo qual promove o efeito anti-reabsorção é desconhecido, entretanto, acredita-se que ele se incorpore à matriz óssea $\mathrm{e}$ seja assimilado pelos osteoclastos, inibindo-os durante a sua ação ${ }^{(3)}$. A esofagite, estenoses esofágicas e sintomas gastrointestinais são efeitos colaterais importantes desta $\operatorname{droga} a^{(1)}$.

Relata-se um caso de paciente que desenvolveu subestenose e ulceração na porção terminal do esôfago e em porção intestinal de anastomose esôfago-entérica.

\footnotetext{
+ Serviço de Endoscopia Digestiva do Endocenter - Unidade do Fígado e Gastroenterologia - João Pessoa, PB.

Aluno da graduação do curso de Medicina do Centro de Ciências da Saúde e Bolsista do Programa Institucional de Bolsas de Iniciação Científica (PIBIC)/ Conselho Nacional de Ciência e Tecnologia (CNPq)/ Universidade Federal da Paraíba.

** Aluna da graduação do curso de Medicina Centro de Ciências da Saúde/ Universidade Federal da Paraíba.

*** Ex-professor Adjunto de Gastroenterologia da Universidade Federal da Paraíba. Chefe do Serviço de Endoscopia Digestiva do Endocenter - Unidade do Fígado e Gastroenterologia, João Pessoa, PB.

Endereço para correspondência: Dr. Waldir Pedrosa Dias de Amorim - Av. Argemiro de Figueiredo 3701/102 - Bessa - 58036-030 - João Pessoa, PB. e-mail: amorim@openline.com.br
} 


\section{RELATO DE CASO}

MNRS, 63 anos de idade, sexo feminino, submetida a gastrectomia total com anastomose em Y de Roux, em virtude de carcinóide gástrico, em dezembro de 1996. Estava fazendo uso de alendronato na dose de $10 \mathrm{mg}$ ao dia para tratamento de osteoporose desde março de 1999. Não referia qualquer sintoma gastrointestinal prévio. Decorrido 1 mês de tratamento (abril de 1999), passou a apresentar disfagia para alimentos sólidos e dores abdominais difusas. Realizou endoscopia digestiva do trato digestivo superior, que revelou subestenose e ulceração na porção terminal do esôfago (Figura 1) e na mucosa entérica anastomosada. O exame histopatológico dos fragmentos de biopsia obtidos definiu a natureza inflamatória benigna das lesões (processo inflamatório inespecífico em atividade associado à lesão ulcerada).

Após a suspensão da droga, houve desaparecimento da dor abdominal, das ulcerações em porção terminal do esôfago e na mucosa entérica. Entretanto, a subestenose esofágica permaneceu visível em controles endoscópicos subseqüentes, tendo a queixa de disfagia para sólidos diminuído apenas de intensidade. Foi submetida a três sessões de tratamento endoscópico dilatador, bem sucedidas. Atualmente encontra-se em bom estado geral, livre de queixas disfágicas ou dores abdominais.

\section{DISCUSSÃO}

O alendronato de sódio é uma droga aprovada pelo FDA (Food and Drug Administration) para prevenção e tratamento da osteoporose em mulheres pós-menopausadas, como alternativa eficaz à terapia de reposição estrogênica, especialmente para aquelas que não podem ou não farão o uso deste hormônio ${ }^{(5)}$. Recomenda-se que a droga seja

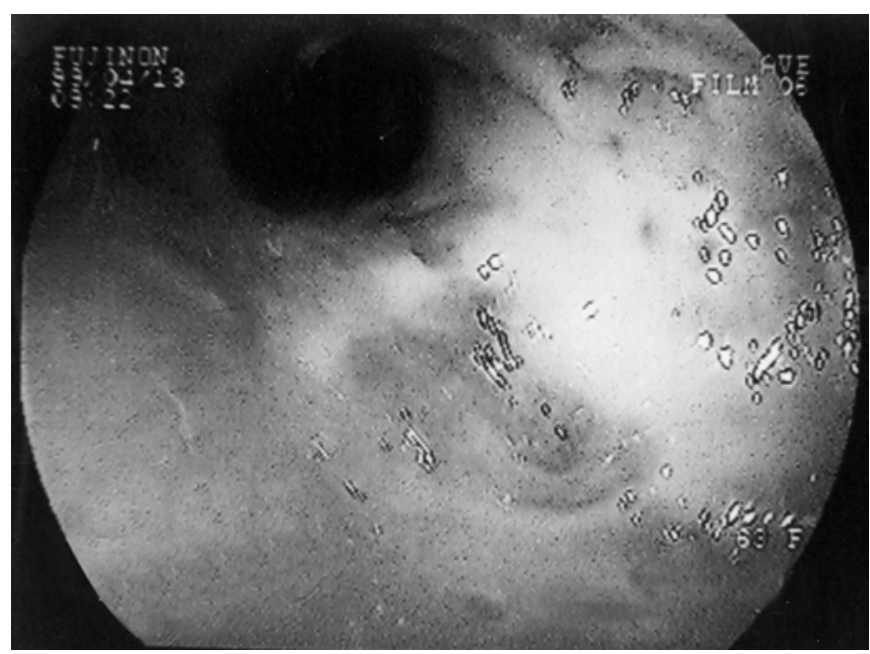

FIGURA 1 - Visão endoscópica de úlcera em anastomose esôfagoentérica tomada pelo menos 30 minutos antes da primeira alimentação ou bebida do dia com um copo cheio de água (180-240 mL), e que se evite deitar ao menos por um período de 30 minutos que sucedem a sua ingestão ${ }^{(2)}$. Essas recomendações visam reduzir o contato do comprimido com o esôfago, durante o seu trânsito pelo órgão e não permitir que o conteúdo gástrico reflua, causando irritação.

MACKAY et al. ${ }^{(4)}$, numa série de 1523 pacientes que apresentavam sintomas associados ao uso de alendronato, verificaram que os principais achados clínicos que levam à descontinuação do tratamento foram dispepsia, náuseas, vômitos e dor abdominal. No presente caso, a disfagia para sólidos e a dor abdominal foram os sintomas que exigiram avaliação endoscópica e descontinuação do tratamento farmacológico. Apesar de estar sendo orientada quanto ao modo das tomadas diárias da droga, a paciente ainda apresentou complicações como subestenose esofágica e ulceração.

Observa-se geralmente que os achados endoscópicos em pacientes com esofagite que fazem o uso de alendronato são compatíveis com agressão química da mucosa esofagiana. Alguns pacientes apresentam erosão ou ulceração com exsudato inflamatório acompanhada de estenose $^{(1)}$. Na paciente em questão, houve lesão na mucosa entérica anastomosada, além da lesão esofágica. Este fato nos leva a questionar a possibilidade do alendronato causar lesões que não no esôfago terminal, despertando cuidados especiais em pacientes gastrectomizados. Aventamos ainda a possibilidade desta droga provocar lesões em segmentos mais baixos do tubo digestivo, como o duodeno, principalmente em pacientes cujo trânsito gástrico seja acelerado.

O envolvimento duodenal, gástrico e o sangramento são achados casuais como complicações do tratamento com o alendronato de sódio. A úlcera esofágica, a esofagite e a esofagite erosiva são os principais efeitos adversos da terapia ${ }^{(1)}$. Para seu controle, a suspensão da droga é mandatória, como procedido no presente caso, e o uso de tratamento ácido-supressor também poderá ser instituído ${ }^{(7)}$. Como complicação temida, a estenose esofágica deve ser sempre considerada durante a avaliação endoscópica. Em algumas situações ${ }^{(6)}$, pode apresentar-se resistente ao tratamento endoscópico dilatador. No presente caso, a paciente foi submetida a três sessões de dilatação endoscópica, denotando melhora progressiva dos sintomas.

Em resumo, acreditamos que o alendronato possa causar lesões tanto da porção inferior do esôfago, como presente em inúmeros relatos na literatura, quanto em segmentos mais baixos do tubo digestivo, em pacientes com trânsito gastrointestinal acelerado. Cuidados especiais devem ser tomados no uso desta droga em pacientes gastrectomizados. A estenose esofágica é complicação que geralmente responde ao tratamento dilatador. Para que tais eventos adversos sejam minimizados, as recomendações quanto à tomada do medicamento devem ser seguidas. A avaliação endoscópica é importante nos casos em que sintomas de irritação esofágica estão presentes e a descontinuação do tratamento é necessária para o seu controle. 
Duques P, Araújo RSA, Amorim WPD de. Esophagus-enteric anastomosis ulceration caused by alendronate. Arq Gastroenterol 2001;38(2):129-131

ABSTRACT - Background - Alendronate sodium is an aminobisphosphonate indicated for the treatment of osteoporosis in post-menopausal women and has been associated with esophagitis in many reports. Esophageal stenosis, gastrointestinal symptoms as dyspepsia, nausea, vomiting and abdominal pain could be present. Objective - Report a case of a patient who underwent total gastrectomy with Y-en-Roux anastomosis for a gastric carcinoid tumor and developed an esophagus-enteric anastomosis ulceration after the use of alendronate. Patient and Method - A 63-year-old woman started medical therapy with alendronate in a dose of $10 \mathrm{mg}$ daily. After a period of one month of medical treatment with this drug she began to complain of dysphagic symptoms and abdominal pain. She was submitted to endoscopic examination that showed an esophageal ulceration, an enteric ulceration of the anastomosis and an esophageal stenosis. Results - Medical treatment with alendronate was discontinued and the symptom of abdominal pain desapeared. The intensity of dysphagia has decreased. The ulcerated lesion remitted although esophageal stenosis did not. The patient was subsequently treated with esophagus-enteric anastomosis dilation. She improved in her general state and nowadays she is free of symptoms. Conclusion - Alendronate sodium could cause lesions of the inferior esophageal portion or in distal segments of the gastrointestinal tube, in patients with a fast gastrointestinal transit. Special attention must be given to gastrectomized patients that use this drug because of the possibility to develop mucosal lesions in the enteric anastomosed part and its fearfull complications as stenosis.

HEADINGS - Alendronate. Esophageal stenosis. Anastomosis, Roux-en-Y. Esophagus, surgery. Enteric ulceration

\section{REFERÊNCIAS BIBLIOGRÁFICAS}

1. De Groen PC, Lubbe DF, Hirsch LJ, Daifotis A, Stephenson W, Freedholm D, Pryor-Tillotson S, Seleznick MJ, Pinkas H, Wang KK. Esophagitis associated with the use of alendronate. N Engl J Med 1996;335:14.

2. Fosamax (alendronate of sodium). Important safety information.- Oesophageal reactions [bula]. Merck Sharp \& Dohme, April 1996.

3. Gilman AG. Fármacos que afetam a calcificação e a renovação do osso. In: Hardman JG, Limbird LE, Molinoff PB, Ruddon RW, editors. As bases farmacológicas da terapêutica. 9. ed. México: McGraw-Hill Interamericana; 1996. p.1139-43.

4. Mackay FJ, Wilton LV, Pearce GL, Freemantle SN, Mann RD. United Kingdom experience with alendronate and oesophageal reactions. Br J Gen Pract 1998;48:429.
5. Ragsdale AB, Barringer TA 3rd, Anastasio GD. Alendronate treatment to prevent osteoporotic fractures. Arch Fam Med 1998;7:6.

6. Ryan JM, Kelsey P, Ryan BM, Mueller PR. Alendronate-induced esophagitis: a case report of a recently recognized form of severe esophagitis with esophageal stricture - radiographic features. Radiology 1998;206:2.

7. Tóth E, Fork FT, Lindelöw K, Lindström E, Verbaan H, Veress B. Alendronateinduced severe esophagitis. A rare and severe reversible side-effect illustrated by three case reports. Lakartidningen 1998;95:35.

Recebido em 5/6/2000 Aprovado em 27/8/2000. 